\title{
La transparencia en la gestión de los recursos naturales. Una mirada regional para transformar la riqueza en desarrollo y evitar la corrupción

Mario Huapaya Nava

Abogado por la Pontificia Universidad Católica del Perú. Maestría en Derecho Empresarial por la Pontificia Universidad Católica del Perú. Máster en Administración Pública por la Universidad de Syracuse.

LL.M por la Universidad Americana. Ex Secretario Técnico del Tribunal de Fiscalización Ambiental del Organismo de

Evaluación y Fiscalización Ambiental.

SUMARIO:

I. Introducción y contexto.

II. Contexto e importancia económica del Sector Extractivo en LAC.

III. ¿Qué factores hacen vulnerable al SE frente a la corrupción y qué actividades de su cadena de valor puede alojarse?

IV. Recomendaciones.

1. Más transparencia y sistemas de rendición de cuentas y menos corrupción.

2. Los principios de Gobierno Corporativo vinculadas a la integridad y sistemas anticorrupción en las empresas del Estado. 


\title{
RESUMEN:
}

Las empresas extractivas —y la gestión de sus ingresos — están particularmente expuestas a actos de corrupción. Los desafíos para que esta industria deje de ser solo una fuente de ingresos económicos para convertirse en generador de desarrollo son enormes, pero no imposibles. Es indispensable que los gobiernos trabajen en proteger y promover su desarrollo de un modo sostenible —y sin corrupción—, para eso la comunidad internacional coincide en que el camino es mejorar las instituciones públicas y fortalecer las relaciones y la confianza de los actores que gobiernan y/o interactúan en el sector. Este documento aborda dos planos de recomendación para ello: la transparencia efectiva de la información, y mejores y más transparentes empresas del Estado del Sector Extractivo.

Palabras clave: Sector extractivo, corrupción, transparencia, gestión de recursos naturales, Latinoamérica y el Caribe

\begin{abstract}
:
Extractive companies, and their revenue management, are particularly exposed to acts of corruption. The challenges for this industry to become a generator of development are enormous but not impossible. Governments must work to protect and promote the development of extractive companies in a sustainable way, without corruption. To achieve that, the international community agrees that improving public institutions and strengthening relationships and trust of the actors that govern and interact in the sector is essential. This paper addresses two recommendations for that goal: information transparency and better and more transparent state companies in the Extractive Sector.

Keywords: Extractive sector, corruption, transparency, natural resource management, Latin America and the Caribbean
\end{abstract}

\section{INTRODUCCIÓN Y CONTEXTO}

No es extraño que los escándalos de corrupción que han sacudido la región en la última década se hayan originado en una empresa de hidrocarburos mayoritariamente estatal - PETROBRAS-. Tampoco que el uso ilegal de fondos producto de la industria minera haya alcanzado a países como Chile, segundo país menos corrupto de la región, por ejemplo, con el caso conocido como Milicolgate. ${ }^{2}$ Las empresas extractivas - y la gestión de sus ingresos- están particularmente expuestas a actos de corrupción.

Un estudio publicado por la $\mathrm{OCDE}^{3}$ respecto a la corrupción en las empresas internacionales, especialmente sobre cohecho - soborno— 427 casos - reveló que el 19\% de los casos sancionados por casos de soborno corresponde a las industrias extractivas, siendo éste el sector más vulnerable a tales hechos, seguido por construcción -15\%-, transporte y almacenamiento - $15 \%$ - e información y comunicación $-10 \%-$. Por otro lado, y refiriéndose específicamente al sub-sector hidrocarburos un estudio de Transparencia Internacional, ${ }^{4}$ en el 2014 identificaba como altamente vulnerable a soborno y otros actos ilegales.

También organismos internacionales de gran influencia como el Fondo Monetario Internacional se han referido al fenómeno y a su pe-

1. “CPI 2020: Américas - News." 2021. Transparency.org. Enero 28, 2021. https://www.transparency.org/es/news/ cpi-2020-americas

2. Mauricio Weibel Barahona. 2015. "Milicogate: El Gran Robo Del Fondo Reservado Del Cobre." The Clinic - Reportajes, Columnas, Entrevistas Y Humor. The Clinic - Reportajes, columnas, entrevistas y humor. August 13, 2015. https://www.theclinic.cl/2015/08/13/milicogate-el-gran-robo-del-fondo-reservado-del-cobre/

3. OCDE, Foreign Bribery Report An Analysis of the Crime of Bribery of Foreign Public Officials (2015) https://read.oecd-ilibrary.org/governance/informe-de-la-ocde-sobre-el-soborno-internacional_9789264226654-es\#page1

4. Transparencia Internacional, Bribe Payers Index (2011) 
ligroso efecto y envergadura en materia fiscal. Basta revisar la inclusión como tema de riesgo en su Informe de Monitoreo Fiscal de abril $2019^{5}$ y su calificación como el cuarto pilar de análisis en el Código de Transparencia Fiscal, ${ }^{6}$ bajo el concepto de Resource Revenue Managment. Para este organismo - como para otros que analizaremos más adelante- la propia naturaleza multinacional y traslado de grandes sumas de dinero e influencias, así como la dependencia de nuestros países, hacen de esta industria especialmente proclive a la corrupción.

Por otro lado, sumado a las posibles y enormes desviaciones ilegales de recursos públicos, y a pesar del gran progreso que han tenido las industrias extractivas en materia de mejores prácticas de participación ciudadana y transparencia, por ejemplo adhiriéndose al Estándar Global para la Transparencia en las Industrias Extractivas - EITI, - y en general mejoras en la gobernanza del sector-, aún persisten en la región índices muy altos de desconfianza entre los actores, misma que en su mayoría viene precedida en muchos casos por una ineficiencia tanto pública como privada a lo largo de toda la cadena de valor de la industria. A saber, en 2017, la región recibió una calificación de 58 puntos sobre 100 del Índice de Gobernanza de los Recursos
Naturales ${ }^{7}$ del Instituto de Gobernanza de los Recursos Naturales - NRGl, que evalúa la gestión de las industrias extractivas en países ricos en recursos naturales. Esta calificación es indicativa de una "débil gobernabilidad de los recursos naturales". ${ }^{8}$ En el $2021^{9}$ este índice ha evolucionado hacia informes por país; los países de la región ADMINISTRATIVO analizados Colombia, Guyana, Perú y México, tampoco alcanzaron buenos resultados. Las calificaciones de Perú por ejemplo en materia del estado de derecho y control de la corrupción tienen calificaciones en promedio de 57/100.

A su vez, la falta de confianza agrava la diferencia de intereses y los torna en conflictos y empeora los ya de por sí inevitables efectos sociales y ambientales negativos de la industria. Se puede citar por ejemplo que el Observatorio de Conflictos Mineros de América Latina - OCMAL, ${ }^{10}$ identifica actualmente 284 conflictos relacionados con la minería, mientras que la Defensoría del Pueblo del Perú11 encuentra en este país a agosto de 2021, 198 conflictos socioambientales se relacionan con el Sector Extractivo - SE.

En este contexto, los desafíos para que una industria tan importante para la región deje de ser solo una fuente de ingresos económicos para convertirse en generador de desarrollo son enor-

5. "Fiscal Monitor:Curbing Corruption." 2021.IMF.2021.https://www.imf.org/en/Publications/FM/Issues/2019/03/18/ fiscal-monitor-april-2019.

6. "El Codigo De Transparencia Fiscal" IMF. https://www.imf.org/external/"np/fad/trans/Code2019.pdf

7. "Índice de La Gobernanza de Los Recursos Naturales 2017." 2017. Natural Resource Governance Institute. July 13, 2017. https://resourcegovernance.org/analysis-tools/publications/indice-de-la-gobernanza-de-los-recursos-naturales-2017.

8. NRGI 2017a; para una revisión de los marcos institucionales de la minería ver Vio y Walter 2016.

9. "Announcing the 2021 Resource Governance Index: Assessments of Oil, Gas and Mining Governance in 18 Countries." 2021. Natural Resource Governance Institute. Junio 14, 2021. https://resourcegovernance.org/blog/2021-resource-governance-index-assessments-oil-gas-mining-governance-in-18-countries.

10. Latina. 2021. “Conflictos Mineros En América Latina." Observatorio de Conflictos Mineros de América Latina, OCMAL. 2021. https://mapa.conflictosmineros.net/ocmal_db-v2/.

11. "195 Conflictos Sociales Esperan Ser Atendidos Por El Gobierno de Pedro Castillo." 2021. Observatorio de Conflictos Mineros de América Latina. August 16, 2021. https://www.ocmal.org/195-conflictos-sociales-esperan-ser-atendidos-por-el-gobierno-de-pedro-castillo/. 
mes, pero afortunadamente no imposibles. Un recuento interesante de países que lo lograron son Australia, Botswana y Canadá, quienes fortaleciendo sus instituciones han vencido la paradoja de la "maldición de los recursos" o "enfermedad holandesa" - Sachs y Warner, 2001. Lane y Tornell 1999_, revertiendo eventualmente sus índices de pobreza, corrupción y conflictos.

En buena cuenta, siendo el SE tan importante para el desarrollo de la región es indispensable que los gobiernos trabajen en protegerlo y promover su desarrollo de un modo sostenible $-y$ sin corrupción-. Para eso la comunidad internacional coincide en que el camino es mejorar las instituciones públicas y fortalecer las relaciones y la confianza de los actores que gobiernan y/o interactúan en el sector.

Este documento aborda dos planos de recomendación, mismos que son validados por múltiples organizaciones y estándares internacionales y que pueden derivar en acciones concretas de políticas públicas en nuestros países:

a) La transparencia efectiva de la información, misma que, una vez adoptada como requisito inicial de las políticas públicas debe tener un rol cada vez más activo, coherente y consustancial a otras políticas públicas. Esta sección incluye una referencia a la necesidad de fortalecer la comunicación constructiva entre los stakeholders del SE y proyectarla a mejorar la rendición de cuentas y la participación y;

b) Mejores y más transparentes empresas del Estado del Sector Extractivo, bajo principios de Gobierno Corporativo y especialmente en el caso de las empresas petroleras estatales y semi estatales.

II. CONTEXTO E IMPORTANCIA ECONÓMICA DEL SECTOR EXTRACTIVO EN LATIONAMÉRICA Y EL CARIBE

Para entender la importancia y necesidad de profundizar en las reformas y prácticas de fortalecimiento institucional que aseguren la sostenibilidad del SE en LAC, es necesario repasar su importancia económica actual y potencial, así como su evolución y los distintos escenarios de desarrollo de la industria en los próximos años.

Siendo el SE altamente dependiente de la fluctuación de los precios, es posible identificar hasta tres grandes momentos o contextos económicos en las últimas dos décadas, en todas ellas el impacto de las SE en las economías nacionales es crucial:

a) Una primera etapa entre el 2003 y 2014 marcada por el alto precio de los metales, llamada comúnmente como del "boom de los comodities". En esta etapa, para los países de la región, las rentas del SE representaron entre el $4 \%$ y el $7 \%$ del PBI e impulsaron la generación de empleo, inversiones públicas y sociales. Según los indicadores de desarrollo del Banco Mundial, en este periodo el SE ayudó efectiva y objetivamente a reducir la pobreza;

b) Una segunda etapa que empezó entre el 2013 y 2014 marcada por el retroceso de los precios y por tanto recorte en los presupuestos y contribuciones de las empresas extractivas, con el consecuente impacto negativo en toda la economía; $y$

c) Una última etapa - actual- cuyo inicio podría encontrarse en el 2016 donde los precios han empezado a recuperarse, dando oportunidad a los países a volver a impulsar sus economías. Según el BID ${ }^{12}$, citando los datos del "S\&P Global Market Intelligence", en minería se reportan una recuperación de la inversión a partir de 2016 y 2017 por ejemplo a partir del crecimiento de la exploración de metales no ferrosos en $15 \%$. Por otro lado, en materia de hidrocarburos a partir de 2016, el BID citando a la International Energy Agency indica que la inversión en exploración y producción también empezó a dar señales de recupe-

12. Vieyra, Aamot \& otros. Gobernanza con transparencia en tiempos de incertidumbre. BID, 2019 
ración, con un crecimiento global de $4 \%$ en 2017 hasta alcanzar USD 450.000 millones, y de 5\% en 2018.

Finalmente, entre el año 2020 y 2021 parece haberse consolidado una franca recuperación de precios tanto de los metales como del petróleo, hasta niveles superiores el boom del numeral (a) anterior. La evidencia más objetiva sería por ejemplo la cotización del cobre que se ha venido recuperando significativamente desde enero del 2016 -1.94 dólares la libra-, y ahora el precio del metal rojo se sitúa en 3.18 dólares la libra, en los mercados internacionales. ${ }^{13}$

Para capitalizar el momento los países que cuentan con reservas probadas de recursos deben elevar su producción. Por ejemplo, en Venezuela, donde la relación reservas-producción es superior a 100 años, o considerando proyectos como la mina Chuquicamata en el norte de Chile - la mina de cobre a cielo a abierto más grande del mundo-o el campo Cantarell en México-con más de 35 billones de barriles en reservas. Por otro lado, en países donde ya la producción es de nivel mundial, asegurar las inversiones y garantizar el desarrollo de todo su potencial. A saber, entre Perú y Chile acumulan el $43 \%$ de la producción mundial de cobre, Chile y Argentina están entre los tres primeros productores de Litio en todo el mundo y en conjunto los países de Latinoamérica y el Caribe - LAC, contribuyen al $9 \%$ de la producción mundial de petróleo y al $6 \%$ de gas natural.
III. ¿QUÉ FACTORES HACEN VULNERABLE AL SE FRENTE A LA CORRUPCIÓN Y QUÉ ACTIVIDADES DE SU CADENA DE VALOR PUEDE ALOJARSE?

La corrupción como en las otras industrias, se comporta como un lastre para la actividad económica, no permitiendo su evolución natural y encareciendo la inversión. La corrupción eleva artificialmente los costos y altera la distribución de los recursos y de las ganancias que estos producen, es decir asigna la riqueza de manera inequitativa e irracional. Desde el punto de vista social y político debilita el sistema y la confianza entre los actores y hace cada vez más difícil la implementación exitosa de políticas públicas.

Luchar contra la corrupción requiere conocerla y tipificarla y para ello es necesario conocer profundamente el sector y cada una de sus intervenciones en la vida económica de los países. El estudio de Mayorga publicado por el Banco Mundial $-2009-{ }^{14}$ sobre la cadena de valor de la industria extractiva, así como los estudios metodológicos de OCDE y el EITI, ${ }^{15}$ reconocen 6 fases consecutivas del SE empezando por (a) la decisión de extraer el recurso (b) la entrega de contratos y licencias (c) la producción del recurso — regulada y monitoreada - (d) la recaudación de ingresos -impuestos y regalías - (e) la gestión de los ingresos y la asignación de la renta y (f) la inversión o gasto social de los ingresos fiscales.

Los estudios de Transparencia Internacional ${ }^{16}$ y $\mathrm{OCDE}^{17}$ coinciden en que existen factores de

13. FMI Perú regresa a niveles del boom de precios de los metales, acceso el 24 de septiembre de 2021. https://iimp. org.pe/raiz/fmi-peru-regresa-a-niveles-del-boom-de-precios-de-los-metales

14. Mayorga, Eleodoro Extractive Industries Value Chain, World Bank 2009. https://openknowledge.worldbank.org/ bitstream/handle/10986/18400/484240NWP0Box31ei1for1development13.pdf?sequence=1\&isAllowed=y

15. "La Cadena de Valor Del EITI." 2021. Extractive Industries Transparency Initiative. 2021. https://eiti.org/es/cadena-valor-del-eiti.

16. "Combatting Corruption in Mining Approvals: Assessing the Risks in 18...." 2017. Transparency.org. December 5 , 2017. https://www.transparency.org/en/publications/combatting-corruption-in-mining-approvals.

17. "CORRUPTION in the EXTRACTIVE VALUE CHAIN: TYPOLOGY of RISKS, MITIGATION MEASURES and INCENTIVES." n.d. Accessed October 24, 2021. https://www.oecd.org/dev/Corruption-in-the-extractive-value-chain.pdf. 
riesgo de corrupción en cada una de las fases de la cadena de valor, exacerbados principalmente por factores como los siguientes:

a) Existe debilidad en los sistemas anticorrupción vinculada a los bajos índices de estado de derecho y escasa capacidad institucional para detectar, prevenir y sancionar efectivamente los actos ilícitos. Esta debilidad viene precedida por insuficientes fuentes de información exacta y actualizada sobre reservas, permisos y resultados de la explotación.

b) Excesiva discrecionalidad entre los oficiales públicos que deciden incorporar zonas de exploración o explotación, así como facultades amplias para decidir los procesos de licenciamiento.

c) Exposición de los oficiales a influencias políticas, económicas, posible captura por parte de intereses privados y rotaciones público-privadas - puerta giratoria-. Situación que se agrava al incluir en la esfera de decisión a gobiernos subnacionales, bajo cuya jurisdicción pueden encontrarse las minas y/o yacimientos.

d) Considerando la alta tecnificación y los lugares remotos de muchos de los procesos extractivos, existe una fuerte dependencia pública y privada de profesionales expertos disponibles, cuya oferta en el mercado es limitada.

e) En el caso de las empresas privadas, débiles o extemporáneas políticas de due diligence y exámenes de integridad de sus cuerpos gerenciales. En el caso particular de las empresas de petróleo E\&Y menciona la alta dependencia al pago en efectivo de servicios y proveedores.

Por su parte el Instituto de Gobernanza de los Recursos Naturales, ${ }^{18}$ refiriéndose al más delica- do de los pasos en la cadena de valor, como es la entrega de licencias y contratos, ha llamado la atención sobre 12 situaciones que deberían disparar las alertas en la sociedad civil y el Gobierno, estas incluyen: permitir participar en procesos competitivos a empresas poco calificadas, no considerar los antecedentes criminales de empresas en competencia o relación con personas políticamente expuestas - PEP, intervención de oficiales relacionados a las empresas en competencia, posibilidad de sobornos, conflicto de intereses, y la posibilidad de que las empresas adjudicatarias vendan los proyectos sin cumplir sus compromisos de inversión.

Finalmente, y considerando todo lo anterior existen para LAC recomendaciones específicas a partir del Informe del Grupo Asesor de Expertos en anticorrupción transparencia e integridad de América Latina y el Caribe ${ }^{19}$ del BID en el sentido en que una correcta gestión - sin corrupción- de los recursos naturales requiere de parte de los Estados:

a) Empezar por un planeamiento estratégico con un enfoque de gobernanza territorial sostenible que permita la gestión organizada de los Recursos naturales a mediano plazo, fortalecimiento de las instituciones públicas responsables y diversificación económica.

b) Evaluación sistemática de las vulnerabilidades de gobernanza y corrupción en toda la cadena de decisión del sector extractivo.

c) Reforma de las empresas del Estado para la implementación de mecanismos efectivos de integridad, mejores competencias y despolitización de las decisiones. En un apartado posterior se indican cuales son esas medidas concretas a partir de los principios de Gobierno Corporativo.

d) Fortalecimiento de instituciones a nivel

18. Sayne, Aaron, Alexandra Gillies, and Andrew Watkins. 2017. "Twelve Red Flags: Corruption Risks in the Award of Extractive Sector Licenses and Contracts." https://resourcegovernance.org/sites/default/files/documents/corruption-risks-in-the-award-of-extractive-sector-licenses-and-contracts.pdf.

19. Informe del Grupo Asesor de Expertos en anticorrupción transparencia e integridad de América Latina y el Caribe, BID 2018. En su sección referida a Gobernanza de los Recursos Naturales. P.14 
subnacional y estándares ambientales y sociales. Instituciones de gobierno, pero también de interrelación con las comunidades para desarrollar juntos planes sobre la base del costo beneficio, y conciliación de intereses, incluyendo el análisis y reducción de conflictos.

e) Mejora de la transparencia y rendición de cuentas en el sector de las industrias extractivas, que se desarrolla en un numeral posterior de este mismo documento

f) Mejora de la gobernanza y eficacia de los fondos soberanos, incluidos los fondos de estabilización y desarrollo intergeneracional, por ejemplo, adoptando estándares internacionales como los Principios de Santiago. Estas soluciones institucionales mejoran por lo general con mejores estructuras de gobierno independientes y controles cruzados entre las autoridades con poder de decisión.

g) Mejores recursos para el monitoreo de contrataciones públicas y transparencia financiera, además de complementar la regulación del sector por ejemplo con la de los beneficiarios reales o finales.

Seguidamente este documento desarrolla dos de estas estas recomendaciones, la relacionada con la transparencia y la que pone como objetivo a las empresas estatales y semiestatales del SE.

\section{RECOMENDACIONES}

1. Más transparencia y sistemas de rendición de cuentas y menos corrupción.

La transparencia es un factor clave en el establecimiento de las condiciones sistémicas que favorecen la buena gobernanza en el SE. La transparencia, que en muchos casos viene aparejada en LAC por leyes de acceso a la información, encuentra su correlato en instrumentos internacionales aprobados como el caso del Acuerdo de Escazú ${ }^{0}$ —aunque no ratificado en todos los países de la región-, además de salvaguardas de instituciones como el Banco Mundial y el BID.

La transparencia fomenta la confianza entre las empresas, los actores de la sociedad civil, los ciudadanos y los gobiernos. Es un factor cen-ADMIIIITRATIVO tral en la rendición de cuentas que permite la identificación de brechas de eficiencia en el uso y asignación de los recursos públicos —Kahn, Baron y Vieyra 2018-.

La transparencia no es un fin en sí mismo, y aunque muchos países han establecido obligaciones legales y se han adherido a estándares internacionales como el EITI no ha sido suficiente para vencer las barreras de la desconfianza, promover un planeamiento territorial participativo y evitar los conflictos socio ambientales.

Para trabajar en soluciones los países deben concentrar sus esfuerzos en mejorar e integrar sus sistemas de información para que esta sea oportuna, de libre acceso y exacta, para esto se sugiere la incorporación de procesos de conciliación multisectorial y la incorporación de soluciones tecnológicas como las propuestas por el BID, el EITI, y otras organizaciones.

Bajo las consideraciones anteriores, la transparencia que necesitan nuestros países sigue y debe seguir "dando la batalla", su potencialidad va más allá de una buena práctica que reciba elogios o certificaciones internacionales. Ser transparente es el punto de partida para identificar las reformas necesarias en el sector público, promoviendo su eficiencia y gatillando el acercamiento entre todas las partes.

La transparencia en las industrias extractivas está, por tanto, en un momento crucial, en el que todos creen y confían en ella y ella debe ofrecer resultados concretos y efectivos y así, legitimarse entre todos los actores. Para eso hace falta concebirla en sus dinámicas públicas

20. Acuerdo Regional sobre el Acceso a la Información, la Participación Pública y el Acceso a la Justicia en Asuntos Ambientales en América Latina y el Caribe - Marzo 2019, Costa Rica 
e impulsarla coordinadamente en los siguientes roles:

\section{a) Transformadora desde y hacia lo subna- cional}

Seguir transparentando información sectorial pero sólo para - $y$ desde- las capitales de nuestros países y con la complejidad que solo pueda ser útil para miembros de la industria o ciudadanos medianamente entrenados, es un ejercicio anodino y hasta ocioso. Peor aún acrecienta la desconfianza y hace tristemente evidente las ya existentes brechas de desarrollo y capacidad institucional entre las administraciones centrales y las de niveles subnacionales.

La transparencia que transforma es la que permite a personas que no tenían información, tener acceso a la misma, entenderla, apropiársela y usarla constructivamente. En la región tal condición de ausencia de esta información se verifica mayoritariamente en las regiones, provincias y localidades, donde justamente se desarrolla las industrias extractivas. Es claro que la "maldición de los recursos" es mucho más profunda en los niveles subnacionales.

Los esfuerzos para revertir esta situación han empezado y aunque parciales son positivos, por un lado, el EITI inició en Perú —el país pionero en la aplicación del estándar- los reportes a nivel Regional con Moquegua, Piura y Arequipa. Por otro lado, el BID apoyó los estudios que se llevaron a cabo en Argentina para lanzar una primera plataforma de información minera que incluye información provincial —Centro de Información Minera Argentina - CIMA-. ${ }^{21} \mathrm{~A}$ esto habría que sumarle que también el BID ha incorporado en el Plan para la Segunda Fase de su Plataforma MAPA INVERSIONES Perúpaísmine- ro ${ }^{22}$ módulos en quechua y aimara, además de hipervínculos a explicaciones auditivas, conscientes de la tradición oral de tales lenguas.

\section{b) Articulada y de adaptación inteligente a cada contexto}

Al ser la transparencia en el sector extractivo una condición, la estrategia para que esa condición sea adecuadamente impuesta empieza por un análisis de la situación de cada país y sobre todo por identificar que quieren lograr sus stakeholders en materia de gobernanza en el corto, mediano y largo plazo.

Solo así, los esfuerzos serán adecuadamente administrados y las conquistas serán sólidas y sostenibles. Para poner un ejemplo, no fue ni será lo mismo implementar y perseverar en iniciativas EITI en Perú o Colombia que en Guatemala o Argentina. Del mismo modo adaptarse al tiempo de cada país en materia extractiva; no es igual de urgente la transparencia en Guyana hoy que antes del descubrimiento de sus reservas de petróleo.

Igual de compleja pero necesaria, es la adaptación que debe exhibir la transparencia para incluir en su ámbito a otro tipo de información no necesariamente extractiva. La transparencia, para ser efectiva, tiene que ser multisectorial, así como lo son los efectos de la industria. Esto requiere de ejercicios de coordinación y alianza estratégica con otros sectores, como los ministerios de economía, ambiente, de prestaciones sociales, empleo y otros.

En este aspecto también se han iniciado ya los cambios estratégicos. El EITI por ejemplo ya contempla en el estándar del 2016 la llamada

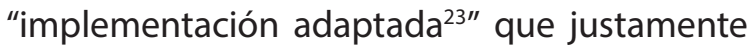
reconoce que no todos los países candidatos

21. Véase en: http://cima.minem.gob.ar/dataset/420

22. "Consulta Los Proyectos Y Recursos En Mapa Inversiones." 2012. Minem.gob.pe. 2012. https://mapainversiones. minem.gob.pe/\#/proyectos.

23. "The EITI Standard 2016." 2016. Extractive Industries Transparency Initiative. March 2, 2016. https://eiti.org/document/eiti-standard-2016\#r8. 
son iguales. Por parte del BID y su ya citada plataforma MAPA INVERSIONES Perúpaísminero, ${ }^{24}$ ya incluye información sobre el empleo generado por la minería y en sus fases siguientes se espera colocar compromisos ambientales y sociales. La idea de fondo es que la transparencia sea una herramienta adaptada y útil y no solo una lista de información disponible.

\section{c) De vanguardia tecnológica y con proyec- ción a la sociedad civil}

Relacionada, pero distinta a la necesidad de adaptación, es la manera en que la información es puesta a consideración de los ciudadanos. Aplicar tecnología para este propósito implica decidir el mejor conjunto de instrumentos, recursos técnicos y procedimientos para que la información realmente comunique.

En el caso de las industrias extractivas, como en muchos otros campos, claramente el espacio está ocupado por la información entregada a demanda y permanentemente en línea y actualizada. Por eso el EITI ha pasado de informes físicos a información online - portales de transparencia-y los gobiernos optan por fortalecer sus gobiernos abiertos y digitalizados.

Sin embargo y aunque necesariamente tecnológica la transparencia requiere considerar las condiciones de los receptores de la información, incluyendo su acceso a la internet, el idioma, el nivel y su capacidad de entender e interpretar los datos. El horizonte es claramente cerrar las brechas en el mediano plazo y volcarse a una participación activa de la sociedad civil en sus desarrollos territoriales. Claro ejemplo de esa visión se recoge en la recientemente lanzada Visión de la Minería al $2030^{25}$ en el Perú que propugna una minería inclusiva e integrada social y territorialmente.

\section{d) Fomentar la Transparencia en materia de beneficiarios finales}

Uno de los temas de mayor relevancia en el SE y vinculado íntimamente con la transparencia, es el relacionado con la prevención y el control de la corrupción vía el registro y divulgación de los beneficiarios finales, definidas como aquellas personas naturales que ejercen control efectivo o detentan la posesión sobre un cliente en cuyo nombre se realiza una transacción, o que ejercen el control efectivo final sobre una persona jurídica u otra estructura jurídica - GAFI, 2012-.

La identificación de los beneficiarios finales es un instrumento fundamental para mitigar riesgos de corrupción en el SE, permitiendo levantar el velo de anonimato e identificar a los beneficiarios finales de una empresa o de una licencia de explotación de un recurso minero o hidrocarburiferos -BID 2018-.

En el sector extractivo es importante identificar los riesgos asociados a los beneficiarios reales a lo largo de la vida de un proyecto, por ejemplo: (i) al asignar derechos la participación oculta de personas expuestas políticamente puede elevar el riesgo de corrupción en la licitación y la contratación; (ii) en la fase operativa, se limita la rendición de cuentas por impactos ambientales o sociales negativos, o debilitar las políticas existentes para promover la creación de valor local; y (iii) en la administración de ingresos, las compañías pueden usar transacciones entre entidades relacionadas - vía precios de transferencia- para evadir impuestos.

\section{Los principios de Gobierno Corporativo vinculados a la integridad y sistemas an- ticorrupción en las empresas del Estado.}

Aunque existen múltiples mandatos para que las empresas de propiedad del Estado de LAC

24. "Consulta Los Proyectos Y Recursos En Mapa Inversiones." 2012. Minem.gob.pe. 2012. https://mapainversiones. minem.gob.pe/\#/proyectos.

25. “Rimay Culmina La Elaboración de Documento: 'Visión de La Minería al 2030."' 2019. www.gob.pe. 2019. https://www.gob.pe/institucion/minem/noticias/25008-rimay-culmina-la-elaboracion-de-documento-vision-de-la-mineria-al-2030. 
se acojan a los principios internacionales de Gobierno Corporativo, por ejemplo, los impulsado por la OCDE - 2015 - ${ }^{26}$, muchos de ellos no han desarrollado mecanismos específicos y efectivos que les permita autoevaluarse y fijarse metas en cuanto a la transparencia, control y rendición de cuentas. Como consecuencia y sujetas a grandes presiones, se han verificado en ellas problemas graves de corrupción, a lo largo de la vida económica de las empresas.

En base a los estudios de E\&Y y OCDE los principales factores de corrupción se concentran en este tipo de empresas considerando factores como (i) sus operaciones comerciales implican grandes sumas de dinero, lo cual hace atractivo intereses de toda índole $-\mathrm{y}$ expuestas a corrupción - sus operaciones de compra y adquisiciones, acuerdos asociativos, y cadena de proveedores; (ii) las actividades petroleras se desarrollan en zonas generalmente apartadas y de difícil acceso, lo cual dificulta la supervisión y control (iii) los controles de las empresas muchas veces se agotan en revisión formal de documentos y no en control de gestión o de transacciones comerciales y (iv) pueden existir influencias políticas en las decisiones empresariales, incluyendo las decisiones de contratación de gerentes y el diseño de pesos y contrapesos de los órganos de control de las empresas.

Respecto a las recomendaciones para este tipo de empresas, además de los principios de transparencia existen otras fuentes como, por ejemplo: Los 10 principios Anti-Corrupción para empresas públicas -2017- de Transparencia Internacional, la Guía sobre transparencia de las empresas extractivas de propiedad estatal -2018 — NRGI - y las Reglas para la publicación de información relacionada a pagos realizados por las industrias extractivas —2013 Unión Europea-.

Entre las recomendaciones referidas a la necesidad de divulgar información y potenciar su utilización, una rendición de cuentas rigurosa y establecer la integridad se encuentran:

a) Impulsar la accesibilidad a información de manera oportuna, clara y abierta, tratando por ejemplo de alcanzar los estándares de las empresas que cotizan en el mercado de capitales. Para completar esta recomendación es necesario que la información se disponga en formatos que permitan la inteligencia de datos y los reportes a cada tipo de stakeholders de las compañías.

b) La información debe ser completa y exacta respecto al comportamiento económico y de decisiones de la empresa. Los órganos de gobierno de la sociedad deben ser responsables de la misma y supervisar su producción. Las excepciones deben ser justificadas y aprobadas por el órgano más alto de la empresa.

c) La información debe contener una necesaria referencia a cómo está organizada y gobernada la empresa, así como sus relaciones con empresas subsidiarias, filiales y en Joint Venture a todo nivel -incluyendo especialmente la existencia de subsidios-. Si se trata de empresas mixtas es relevante que la transparencia alcance a que se conozcan los reales propietarios o beneficiarios reales.

d) Los procesos de adquisiciones de bienes y contratación de servicios deben anunciarse, así como las reglas de competencia y evaluación de los candidatos.

e) La rendición de cuentas a los stakeholders debe ser oportuna, regular y relevante. Este ejercicio no solo debería incluir los reportes financieros obligatorios sino todos a los que se comprometa como parte de una gestión constructiva y transparente de la empresa.

f) Se deben establecer mecanismos de divulgación de los resultados de los ejercicios de compliance legal, ambiental, social, etcétera, que permitan tanto a las autoridades de la empresa como a los Estados conocer el verdadero estado de los casos y las auditorías que se realicen.

26. Directrices sobre el Gobierno Corporativo en las Empresas Públicas, publicadas por la Organización para la Cooperación y el Desarrollo Económico - OCDE 